\title{
VII. SCHLUSSBETRACHTUNG
}

Denn nachdem die Bibel ins Englische übersetzt worden war, glaubte jedermann, ja sogar jeder Junge und jedes Mädchen, die lesen konnten, sie sprächen mit Gott dem Allmächtigen und verstünden, was er sagte, wenn sie eine Anzabl von Kapiteln pro Tag aus der Heiligen Schrift ein-oder zweimal gelesen hätten. Damit wurden die Ehrfurcht und der Gehorsam der reformierten Kirche gegenüber den Bischöfen und der Geistlichkeit darin vernichtet, und jeder wurde jetzt selbst Richter der Religion und ein Ausleger der Heiligen Schrift für sich selbst.

(Новbes, Behemoth oder das Lange Parlament, S.30)

In ähnlicher Weise [wie die Pädagogen] beanspruchen die öffentlichen Sittenlebrer und Gottesgelehrten, die Könige selbst und die höchsten Herren der Kirche zu leiten, obne $z u$ wissen, von wem sie zu so einer bedeutenden Aufgabe berufen werden; da sie vielmehr den Glauben erwecken möchten, nicht den Königen und Männern, deren Sorge Gott das Wobl des Volkes anvertraut hat, sondern ibnen selbst sei diese Aufgabe von Gott unmittelbar übertragen zur großen Gefabr für den Staat.

(Hobbes, Lehre vom Menschen, S.43)

Für Thomas Hobbes waren die Ursachen des englischen Bürgerkrieges wesentlich geistiger Natur. Bibel und antike Philosophie waren für ihn subversive Arsenale, aus denen die Kritiker der Monarchie ihre Argumente speisten. Kirchen und Universitäten sah er als Orte, in denen die Untertanen und die Mitglieder der politischen Führungsschicht Werte und Vorbilder vermittelt bekamen, die ihre Loyalität zur Krone untergruben. Sofern es nicht gelang, den Zugriff auf die Speicher und Autoritätsquellen politischer Sprechakte zu domestizieren und die Auslegung dieser Quellen qua politischer Autorität vorzugeben, war die Monarchie in Hobbes Augen auf Sand gebaut. Hobbes war keineswegs der einzige, der das potentiell herrschaftsgefährdende Potential der Bibel zur Sprache brachte. Nach dem Zusammenbruch der etablierten Kirchenordnung in den Herbstmonaten des Jahres 1640 klagten einige Conformists in der englischen Kirche über die politischen Folgen der Tatsache, daß die Bibel in englischer Sprache von jedermann zu Rate gezogen werden könne. ${ }^{1}$ Das Gotteswort war in ihren Augen eine politische Ressource, die sich der Kontrolle der kirchlichen ebenso wie der weltlichen Obrigkeit entzog und deren Autorität schwinden ließ.

Vor dem Hintergrund der zeitgenössischen Erfahrungen waren diese Klagen des hohen Klerus verständlich. Allerdings lassen sowohl Hobbes als auch die Conformists in ihrer Beschwerde über die subversiven Folgen der allgemein ver-

1 Beispiele finden sich in Cressy, England on Edge, S. 266. 
fügbaren Heiligen Schrift unerwähnt, daß es nicht nur die Kritiker von König und Klerus waren, die sich der politischen Ressource der Bibel bedienten. Der Rückblick bis zum Beginn der „politischen Reformation“ Heinrichs VIII. hat gezeigt, daß zahlreiche Akteure in Staat und Kirche sich der Autorität der Bibel bedienten, um aus ihr Legitimität für den eigenen Statusanspruch zu gewinnen oder konkrete politische Forderungen aus ihr abzuleiten. Dies läßt sich schon an der vorliegenden Vielzahl englischer Bibelübersetzungen bis zum Bürgerkrieg ablesen.

Die Verfügbarkeit der Heiligen Schrift für all diejenigen in England sicherzustellen, die des Lesens kundig waren, war dabei stets nur ein Anlaß für die Entstehung immer neuer Bibelübersetzungen und -ausgaben. Eine weitere Ursache war die Möglichkeit, durch den Akt der Autorisierung einer Neuausgabe der Heiligen Schrift das Gotteswort in einen Sprechakt der autorisierenden Instanz zu verwandeln. Von dieser Möglichkeit machte Heinrich VIII. mit der Great Bible ebenso Gebrauch wie Jakob I. mit der King James Bible; und auch die Bischöfe wollten dem nicht nachstehen und beauftragten ihrerseits eine Bishop's Bible als Textgrundlage für den Gottesdienst in allen Gemeinden Englands. Selbst die Katholiken wollten die Ressource der Bibel nicht einfach den Protestanten überlassen und brachten mit dem Rheims New Testament eine eigene Übersetzung aus der Exilspresse auf den englischen Markt.

Die Übersetzung der Heiligen Schrift bot stets auch die Möglichkeit, auf die Interpretation der Schrift Einfluß zu nehmen, sei es durch die Kommentierung einzelner Textstellen, wie es insbesondere in der Geneva Bible oder im Rheims New Testament praktiziert wurde, sei es durch Botschaften, wie sie im Titelbild oder in den Paratexten vermittelt wurden. Die Autorität der Schrift bedurfte zusätzlich der richtigen Auslegung, um zu einer scharfen Waffe in den politischen Auseinandersetzungen werden zu können. Wenn die Technik des Buchdrucks die private Bibellektüre ermöglichte, sollten gezielte Botschaften im Umfeld der bereitgestellten Übersetzung dazu führen, daß der Leser aus der Heiligen Schrift die „richtigen“ Schlußfolgerungen zog.

Vor dem Hintergrund der seit 1637 in Schottland und seit 1640 in England erodierenden Herrschaftsgewalt des Königs und der Bischöfe mochten deren Anhänger zwar zunehmend der Meinung gewesen sein, daß die Bibel eine wirkungsvolle politische Ressource vorwiegend in der Hand ihrer Gegner war. Diese Auffassung war in den Jahrzehnten zuvor indes nur vereinzelt anzutreffen. Es hat sich vielmehr gezeigt, daß sich in England und Schottland seit der Reformation Grundauffassungen zum Bibelverständnis etablierten, die den politischen Biblizismus in den Folgejahrzehnten begünstigten.

Während der politischen Reformation unter Heinrich VIII. argumentierten zwei so unterschiedliche Protagonisten wie William Tyndale und Stephen Gardiner gleichermaßen mit der lex dei, um damit die Notwendigkeit der Reformation (Tyndale) bzw. die Suprematie des Königs in der Kirche (Gardiner) zu begründen. Das göttliche Gesetz entnahmen beide Autoren den deuteronomischen Schriften des Alten Testaments, deren Aussagen sie beide als zeitlos gültig aner- 
kannten, zumindest sofern sie das moralische Gesetz Gottes bezeugten. Den mit dem Erlösungstod Christi begründeten Gnadenbund verstanden Tyndale und Gardiner und mit ihnen die überwiegende Mehrheit des englischen Klerus als Bekräftigung der im Alten Testament festgeschriebenen lex dei, nicht aber als deren Ablösung. Diese Auffassung von der Einheit beider Testamente machte alle Aussagen im Alten Testament, die sich als Manifestation des moralischen Gesetzes Gottes auslegen ließen, gleichsam zu potentiellen Gesetzestexten, mit denen Institutionen in Staat und Kirche ebenso wie konkrete politische Handlungen legitimiert oder aber delegitimiert werden konnten. Die große Zahl von Sprechakten, in denen sich zahlreiche Akteure seit der Reformation in unterschiedlichen Themenfeldern auf die lex dei beriefen und daraus politische Forderungen ableiteten, war hauptverantwortlich dafür, daß sich in England und Schottland der Biblizismus als eine maßgebliche politische Sprache ausbildete.

Eine weitergehende Bedeutungssteigerung erhielt die lex dei als Argument in der politischen Auseinandersetzung durch den Verweis auf die potentiellen Folgen bei Nichtbeachtung der Befehle Gottes. Sowohl in England als auch in Schottland war dabei die Vorstellung eines konditionierten Bundes vorherrschend, der seit Abraham und Moses das gegenseitige Verhältnis zwischen Gott und den Menschen bestimmte. Gott sagt seinem Volk dabei Sicherheit und Wohlergehen zu, sofern es sich seinen Gesetzen unterwirft und insbesondere jeglichem Götzendienst für immer abschwört. Diese Bundesvorstellung prägte seit der Reformation die Auffassung vom Verhältnis beider Länder zu Gott. Sowohl nördlich als auch südlich des Tweed sah man sich in der Nachfolge des Volkes Israel, was zum einen das Heilsversprechen und die besondere Nähe Gottes implizierte, zum anderen aber auch die Gefahr von Gottesstrafen heraufbeschwor, sollte die lex dei verletzt werden. Die Schriften des Alten Testaments gaben dabei erschöpfend Auskunft über die zerstörerischen Folgen einer Mißachtung des göttlichen Willens. Sie statteten die Mahnredner mit einem reichen Inventar an Drohpotential aus und ermöglichten es allen Sprechern, durch eine Rückschau auf die historia sacra zu Aussagen über die Zukunft des eigenen Landes zu gelangen.

Es gab sowohl in Schottland als auch in England vereinzelt Stimmen, die davor warnten, die Bibel als politisches Gesetz- und Musterbuch zu verwenden und die daher den politischen Biblizismus ablehnten. Zu ihnen zählte der schottische Humanist George Buchanan ebenso wie der englische Theologe Richard Hooker, der versuchte, den normativen Gehalt der biblischen Texte auf den Bereich der Dogmatik einzuschränken. Ihre Skepsis blieb aber weitgehend ungeteilt. Vielmehr waren Maximen und Exempla der Heiligen Schrift stets wichtige Bestandteile der politischen Auseinandersetzung. Für die meisten Autoren enthielt die Bibel auch auf Feldern jenseits der christlichen Glaubenslehre verbindliche Aussagen. Wie diese Arbeit zeigt, kam die Bibel als Argumentationslieferant zum Einsatz, um die äußere Gestalt der Kirche zu debattieren, d. h. die Kirchenverfassung und die Formen des Gottesdienstes. Sie war von entscheidender Bedeutung, um die Verankerung der Königsherrschaft im Naturrecht zu diskutieren. Sie enthielt in den historischen Schriften des Alten Testaments zur Geschichte des Vol- 
kes Israel entscheidende historische Anknüpfungspunkte an das Königtum der Juden, die in der politischen Debatte oft und gerne bemüht wurden. Sie kam zum Einsatz, wenn über Krieg und Frieden gestritten wurde. Sie stellte Argumente bereit, wenn dynastische Eheverbindungen zur Diskussion standen. Und ihre Maximen und Exempla waren im Spiel, als die Steuer- und Abgabenpolitik Karls I. die Gemüter erhitzte.

Diese resümierende Aufzählung der unter Bezug auf biblizistische Argumente debattierten Themenfelder läßt deutlich werden, daß die politische Sprache des Biblizismus sich nicht einfach mit Theologie gleichsetzen läßt. Der Biblizismus war zugleich weniger und mehr als eine Fachsprache theologischer Gelehrsamkeit. So fand die gelehrte Debatte in der Fachwissenschaft um den Bedeutungsgehalt der Schrift, unter Rekurs auf die Patristik und die Lehre vom vierfachen Schriftsinn, in der politischen Auseinandersetzung nur im Ausnahmefall ihren Niederschlag. Der Fall des jungen Theologiestudenten John Knight in Oxford hat deutlich werden lassen, wie aus einem theologischen Diskurs - David Pareus' Bibelkommentar über den Römerbrief des Apostels Paulus - erst im Moment der öffentlich vorgetragenen Predigt ein politischer Sprechakt wurde, der weitreichende Reaktionen der Obrigkeit auslöste. Erst der politische Sprechakt hat Sanktions- und Zensurmaßnahmen erforderlich gemacht und die Obrigkeit dazu veranlaßt, sich der theologischen Fachdebatte anzunehmen. Umgekehrt waren biblizistische Sprechakte nicht auf Theologen beschränkt. Auch zahlreiche Juristen des römischen Rechts, Literaten und nicht zuletzt König Jakob VI./I. nutzten diese Sprache, um mit ihr politische Aussagen zu begründen.

Sicherlich läßt sich die politische Sprache des Biblizismus genau wie andere politische Sprachen idealtypisch mit einem Ort verknüpfen, an dem diese Sprechakte besonders prominent angesiedelt waren. Die Sprache des common law war neben den Gerichtshöfen insbesondere im Parlament beheimatet. Das römische Recht fand vor allem in den dafür zuständigen Gerichtshöfen Verwendung, z.B. dem Gerichtshof der Admiralität oder der High Commission. Die antike Staatsphilosophie und der sich daraus speisende civic humanism waren vor allem an den Universitäten des Landes zu Hause. Der primäre Ort des Biblizismus war die Kanzel. Die Auslegung der Heiligen Schrift durch einen fachkundigen Sprecher für ein größeres Publikum und die damit einhergehende Adaption der biblischen Aussagen auf einen aktuellen Kontext war gleichsam der idealtypische Sprechakt in der politischen Sprache des Biblizismus. Diese Zuordnung verschiedener politischer Sprachen zu spezifischen Orten ihrer Verwendung ist eine schematische Beschreibung für den imaginierten „Normalfall“. Um die Dynamik politischer Auseinandersetzungen und die sich daraus ergebenden Folgen zu verstehen, sind aber gerade die Momente der Überschreitung der imaginären Grenzlinien von ausschlaggebender Bedeutung.

So läßt sich anhand zahlreicher biblizistischer Sprechakte bilanzieren, daß die mit der Exegese einhergehende Sprecherrolle eines Schriftkundigen auf der Kanzel weit über den Kreis der Geistlichen hinaus attraktiv war. Diese spezifische Art, politische Aussagen zu treffen, ließ den Sprecher teilhaben an der Autorität 
der Heiligen Schrift. Und sie verlieh ihm eine besondere Autorität, die Bedeutung der Schrift für die Zeitgenossen zu vermitteln. Diese Autorität vermittelte den Anschein, ebenso wie diejenige der Heiligen Schrift von Gott verliehen zu sein, der Sprecher hatte daher Anspruch auf Gehör und Beachtung. Es verwundert nicht, daß diese Sprecherrolle in Schottland und England seit der Reformation eine umstrittene Ressource darstellte.

Das Recht zu predigen gehörte neben dem Jurisdiktionsrecht zu der offiziellen Amtsgewalt der Kirche. In der schottischen Kirk hatte sich dabei spätestens seit dem Second Book of Discipline im Jahr 1578 eine besonders stolze Tradition der freien Predigt ausgebildet: Nur Geistliche hatten das Recht, auf autorisierte Art und Weise die Heilige Schrift öffentlich auszulegen. Weder Bischöfen noch dem König war es erlaubt, Predigten einer Zensur zu unterziehen, auch nicht im Falle direkter Herrschaftskritik. Dieses Recht kam nur der Kirche als Ganzes zu, die sich in ihren regelmäßig tagenden General Assemblies dieser Aufgabe annahm, allerdings oftmals ohne auf die Interessen des Königs Rücksicht zu nehmen. Die Bibel war die Meßlatte, anhand derer die Politik des Königs einer regelmäßigen Prüfung unterzogen wurde, die Exegese das öffentliche Richtschwert, das im Falle von Kritik regelmäßig zum Einsatz kam. Diese Erfahrung veranlaßte Jakob dazu, zum einen selbst das Potential der Exegese für eigene Sprechakte zu nutzen, zum anderen gegen den Widerstand der Presbyterianer die bischöfliche Jurisdiktionsgewalt und die High Commission auch in Schottland zu etablieren - ein Unterfangen, das von Teilen des Klerus als Verrat an der Reformation empfunden und nach der Etablierung des National Covenant alsbald rückgängig gemacht wurde.

In England zählte das Predigen zwar ebenfalls zu den Amtsaufgaben jedes Pfarrers. Er unterstand aber der Jurisdiktionsgewalt seines Bischofs bzw. der High Commission als innerkirchliches Disziplinargericht. Und da die Bischöfe vom König ernannt wurden und er als Supreme Head of the Church eine Aufsichtsfunktion über die Kirche wahrnahm, blieb die Freiheit des Kanzelworts eine Ressource, die an Bedingungen geknüpft war. Die wachsende Kritik an Jakobs Friedenspolitik im Dreißigjährigen Krieg und seinen dynastischen Heiratsplänen mit Spanien veranlaßte den König gleich mehrfach dazu, die Geistlichen in England an die Grenzen ihrer Redefreiheit zu erinnern. Diese Maßnahmen zeigten jedoch zugleich, wie schwer es war, zwischen Bibelexegese und politischer Kritik eine eindeutige Grenze zu ziehen. Das schärfere Vorgehen von Karl I. und Erzbischof Laud gegen unliebsame Prediger erweckte bei manchen Zeitgenossen zunehmend den Anschein, gegen die Wahrheit des Gotteswortes selbst gerichtet zu sein und führte dazu, die Bischöfe in ihrer Rolle als Zensoren der Kanzel und das Gericht der High Commission in Augen vieler Protestanten zu delegitimieren.

Dieser Effekt verschärfte sich noch dadurch, daß es anderen Geistlichen gelang, sich selbst auf charismatische Weise als Propheten Gottes zu stilisieren und damit gegen die kirchliche Hierarchie die gleiche Autorität und Unantastbarkeit für sich einzufordern wie ihre alttestamentlichen Vorläufer. Am Beispiel Henry Burtons ließ sich zeigen, wie die beanspruchte Prophetenrolle einzelner Geistlicher die 
Hierarchie der Kirche herausforderte und vor die Wahl stellte, subversive Sprechakte tatenlos zu dulden oder aber mit Strafmaßnahmen gegen Personen vorzugehen, denen zumindest ein Teil der Gläubigen eine besondere Nähe zu Gott zubilligte. Dabei waren die beiden Mitstreiter Burtons auf dem Richtplatz, John Bastwick und William Prynne, keine Geistlichen; gleichwohl reklamierten sie in ihren kritischen Schriften gegen das englische Kirchenregiment die Prophetenrolle mit einigem Erfolg für sich. Die Rückkehr Burtons und seiner Mitgefangenen in die Freiheit verschaffte ihnen darüber hinaus die Möglichkeit, die Rolle der beiden Zeugen der Apokalypse für sich zu beanspruchen und sich damit zu einer wichtigen Figur bei der Erfüllung der Heilsgeschichte zu stilisieren. Diese Inszenierungen waren keine bloße Kuriosität in der Auseinandersetzung um die Kirchenpolitik in England, sondern vielmehr äußerst erfolgreiche Imagepolitik, die den soeben noch verfolgten Sprechern für ihre kirchenpolitische Agenda sowohl Aufmerksamkeit als auch Akzeptanz verschaffte. Im Long Parliament stand für die überwiegende Mehrheit des Unterhauses außer Frage, wer im Falle der Personen Henry Burton und dem Erzbischof von Canterbury, William Laud, zu Recht und wer zu Unrecht Gott für seine Sache reklamierte.

Die protestantische Tradition tat das ihre, um den reklamierten außerordentlichen Sprecherrollen von Burton und seinen Gesinnungsgenossen zusätzliche Legitimität zu verleihen. Reformer wie William Tyndale stellten sich mit ihrem Kampf für den „wahren“ Glauben und gegen den „Götzendienst“ gleichfalls gegen die etablierte Amtskirche und bezahlten dieses Engagement mit ihrem Leben, was ihnen die Aufnahme in das Pantheon protestantischer Erinnerungskultur in England bescherte: eine prominente Erwähnung in John Fox monumentalem Book of Martyrs. In Schottland spielte John Knox eine vergleichbare Rolle, als er sich unerschrocken Maria Stuart entgegenstellte und in der Pose eines zweiten Propheten Jeremias auf die Notwendigkeit einer vollständigen Reformation in Schottland beharrte. Auch diese Sprechakte waren im kollektiven Gedächtnis des schottischen Klerus gespeichert und lieferten ein Vorbild für den unerschrockenen Kampf gegen den Götzendienst. Diese Beispiele verdeutlichen, wie sich die Tradition der Propheten des Alten Testaments dazu nutzen ließ, eine besondere Nähe zu Gott für sich zu reklamieren und damit außerordentliche Sprecherrollen in der Kirche zu legitimieren.

Es ließ sich zeigen, daß auch der junge schottische König Jakob VI. nicht darauf verzichten wollte, sich als oberster Exeget seines Landes zu profilieren, um damit neben seiner Expertise als Theologe auch eine Prophetenrolle für sich zu reklamieren. Er stellte sich selbst sowohl in seinen Psalmenübersetzungen als auch in seiner Meditatioun über Davids Tanz vor der Bundeslade in die Tradition des ruhmreichen David, betonte dabei aber stets die Rolle des Propheten, weniger die des Königs. Seine frühen exegetischen Schriften dienten Jakob dazu, seine Autorität in der Kirche unter Beweis zu stellen, ohne jedoch das presbyterianische Kirchenestablishment offen herauszufordern. Es war dabei eine bewußte Grenzüberschreitung, daß er diese Schriften in der Form von Predigten abfaßte und damit eine Sprecherrolle einnahm, die eigentlich Geistlichen vorbehalten blieb, 
ein Monopol, das die schottischen Presbyterianer besonders energisch verteidigten. Diese zunächst in Schottland erprobte Rolle behielt Jakob über weite Strekken seiner Regierungszeit bei. Dabei nutzte er die spezifischen Aussagemöglichkeiten der Exegese für vielfältige politische Zwecke: Er betrieb mit seinen Schriften Außenpolitik mit England unter Elisabeth I., er untermauerte mit einer Schrift zum divine right of kings seine Erbansprüche auf den englischen Thron, er inszenierte sich in einem Fürstenspiegel an seinen Sohn Heinrich als Herrscher in besonderer Verantwortung gegenüber Gott. In mehreren Schriften wies Jakob den Anspruch des Papstes auf Herrscherabsetzung im Falle einer Exkommunikation zurück und mischte sich darüber hinaus in derselben Angelegenheit auch in eine Debatte der französischen Generalstände ein. Damit stellte er in all diesen Schriften unter Beweis, daß seine Rolle als König sich nicht allein auf weltliche Fragen beschränkte, sondern er auch in geistlichen Angelegenheiten die oberste Autorität verkörperte. Seine Prophetenrolle verschmolz dabei zusehends mit seinem Königsamt. Spätestens als er den englischen Thron bestieg und sich nunmehr als „his sacred majestie“ titulierte, war die Priester- und Prophetenrolle in seiner Selbstdarstellung nicht mehr eine persönliche Qualität, sondern Teil der monarchischen Herrschaftsrolle. Seine Hofkapläne taten das ihre, um daraus eine besondere Unantastbarkeit des Königs abzuleiten und die besondere Nähe des Königs zu Gott zu proklamieren.

Insbesondere in seinen frühen exegetischen Schriften machte sich Jakob zunutze, daß er in der Sprache des Biblizismus über zweierlei Modi des Sprechens verfügte. Im Gegensatz zu seinem Traktat über die Erbmonarchie, The True Lawe of Free Monarchy, in dem er offen die Unantastbarkeit des Königs beschwor und darauf pochte, sowohl in weltlichen als auch in kirchlichen Fragen die oberste Entscheidungsgewalt innezuhaben, ließ er diesen Anspruch in seinen frühen Schriften nur implizit einfließen, indem er biblische Figuren wie König David in den Vordergrund stellte, ohne den Anspruch auf die Führungsrolle in der Kirche ausdrücklich zu erheben. Es blieb dem Leser überlassen, aus den angeführten biblischen Exempla die sich aus ihnen für die eigene Zeit notwendigen politischen Konsequenzen zu ziehen und die Autorität Davids auf Jakob zu übertragen. Der schottische König nutzte mit dieser Möglichkeit des indirekten Sprechens mittels biblischer Exempla eine Argumentationstechnik, die sich ansonsten insbesondere zahlreiche Kritiker der Politik Jakobs I. und Karls I. zu eigen machten, um mit Hilfe biblischer Exempla zwar die gewünschten Assoziationen über die politischen Zustände auszulösen, diese aber nicht direkt zu kritisieren.

Der Kampf um den legitimen Zugriff auf das in den biblischen Schriften gespeicherte Reservoir an Maximen und Exempla setzte sich in Auseinandersetzungen um die richtige Auslegung der einzelnen Bibelstellen fort. Dabei hat die Analyse zahlreicher, äußerst unterschiedlich gelagerter biblizistischer Sprechakte erkennen lassen, daß es sinnvoll ist, bei der Erforschung der politischen Sprache des Biblizismus drei Ebenen voneinander zu unterscheiden:

1. Die Bibel in ihrer Gesamtheit aller kanonischen Schriften stellte das Argumentationsreservoir. Als geheiligtes Gotteswort waren alle in ihr enthaltenen 
Maximen und Exempla gleichsam mit dem Siegel göttlicher Normativität versehen, aller Heterogenität und Widersprüchlichkeit der in ihr enthaltenen Texte zum Trotz. Sie nahm dabei die Rolle eines fundierenden Textes ein, auf den oftmals Bezug genommen wurde, um politische Argumente mit ihr zu autorisieren.

2. Der Zugriff auf die Bibel erfolgte in der politischen Debatte stets selektiv. Es waren einzelne Maximen und Exempla, einzelne in ihr enthaltene Themenstränge und Mustererzählungen, aus denen die Sprecher und Autoren in den hier untersuchten Diskussionen ihre Argumente bezogen. Drei davon spielten in der vorliegenden Untersuchung eine herausgehobene Rolle: Äußerungen zur lex dei, zur Königsherrschaft und der apokalyptische Diskurs vom Untergang Babylons als bevorstehende Ankündigung des Weltenendes.

Das Gesetz Gottes war prominenter Gegenstand der Mehrheit der hier untersuchten Sprechakte. Was dabei unter der lex dei jeweils verstanden wurde, welche Maximen und Exempla jenseits des Dekalogs darüber Auskunft zu geben vermochten und welche Konsequenzen man daraus zur Beurteilung der Politik in Schottland und England zog, hing von der Sinnzuschreibung im Argumentationszusammenhang ab und war nur im Ausnahmefall in den biblischen Schriften selbst präfiguriert. Dies gilt ebenso auch für die unterschiedlichen Aussagen insbesondere aus den historischen Schriften des Alten Testaments zur Monarchie in Israel, die in der Debatte um das divine right of kings bzw. um die Rechte und Pflichten des Königs zahlreich zum Einsatz kamen. Die Ambivalenz der alttestamentlichen Aussagen zum Königtum fand ihr Pendant in der vielseitigen Verwendung dieser Aussagen und Exempla, um je nach Sprecher und politischem Kontext damit die Unantastbarkeit der Könige zu begründen oder aber deren Herrschaftsgewalt einer Reihe von Bedingungen zu unterwerfen. Der Untergang Babylons wurde in zahlreichen Schriften als Warnung an die Zuhörer adressiert, um die Dringlichkeit einer religionspolitischen Kurskorrektur anzumahnen, konnte aber ebenso dazu dienen, den heilsgeschichtlichen Auftrag der Könige in Erinnerung zu rufen, für die bei der Vernichtung Babylons in Johannes' Offenbarung eine entscheidende Rolle vorgesehen sei, woraus z. B. Jakob I. ihre Unantastbarkeit ableitete.

3. Die verschiedenen Mustererzählungen, die in der politischen Debatte regelmäßig aufgegriffen und fortgeschrieben wurden, enthielten ihrerseits nicht jeweils eine in ihnen festgeschriebene Bedeutung, sie standen für eine Vielzahl möglicher Interpretationen offen. In den hier untersuchten Sprechakten kamen sie daher auf sehr unterschiedliche Weise zum Einsatz. Die jeweils bemühten Mustererzählungen waren daher keine politischen Programme einer bestimmten Gruppe in Staat oder Kirche. Sie sind nicht als Ideologie einer bestimmten Gruppe wie der „Puritaner" mißzuverstehen. Selbst der Verweis auf die Apokalypse hatte nicht immer die Funktion, die bestehende Ordnung in Frage zu stellen. Vielmehr waren alle drei hier behandelten Narrative für jeden Sprecher prinzipiell verfügbar, um daraus politische Argumente abzuleiten.

Gleichwohl war der Zugriff auf die in der Bibel enthaltenen Mustererzählungen nicht beliebig. Insbesondere anhand der Imagepolitik sowohl Jakobs VI./I. 
als auch Karls I. hat sich gezeigt, welche Konsequenzen es haben konnte, wenn Könige biblische Maximen zum Maßstab ihres Handelns erklärten oder sich selbst in die Tradition biblischer Vorbilder stellten. Jakobs öffentlich betriebenes Engagement als oberster Exeget im Spiegel seiner Schriften hatte zur Folge, daß er während seiner gesamten Herrschaftszeit auf Aussagen und Rollenbilder festgelegt wurde, die er zu Beginn seiner Herrschaftszeit aus politischen Opportunitätserwägungen heraus getätigt hatte: Seine Selbstbeschreibung als godly ruler, der seine in der Johannesoffenbarung beschriebene Rolle als Kämpfer gegen Babylon und den Antichristen ernst nahm, machte ihn in späteren Regierungsjahren angreifbar, als er sich zu einer Friedenspolitik mit den katholischen Mächten entschloß. Seine öffentlich vorgelegte Interpretation der Apokalypse samt damit einhergehender Handlungsempfehlungen ließ sich nicht vereinbaren mit seiner aktuellen Politik.

Eine ähnliche Erfahrung sollte auch Karl I. machen, als er sich entschloß, im Dreißigjährigen Krieg an der Seite der Protestanten gegen Spanien in den Krieg zu ziehen. Zur Legitimierung dieses Krieges griff Karl auf die lex dei zurück und leitete aus dem Alten Testament die Notwendigkeit ab, bedrängten Glaubensbrüdern beizustehen und deren Kontrahenten als Götzendiener und Gottesfeinde zu bekämpfen. Er griff damit auf ein Rollenverständnis der Königsherrschaft zurück, auf das die zahlreichen Kritiker der Friedenspolitik Jakobs I. rekurrierten, um dessen Friedenspolitik zu brandmarken und um den König auf die Rolle eines Glaubenskämpfers festzulegen. Sicherlich bot sich Karl die Chance, durch eine Adaption dieser Rolle den Kritikern den Wind aus den Segeln zu nehmen. Die Rolle eines Vorkämpfers für den wahren Glauben und gegen den Götzendienst ließ sich aber nicht partiell einnehmen, sondern implizierte, sollte sie glaubhaft sein, weitreichende politische Maßnahmen nicht nur gegen Spanien, sondern auch in der Kirchenpolitik, in der Heiratspolitik und im Umgang mit den englischen Katholiken. Da Karl I. weder bereit noch in der Lage war, diese Agenda vollständig zu erfüllen, verkehrte sich seine an biblischen Rollenvorbildern orientierte Selbstdarstellung in den Augen seiner zahlreichen Kritiker ins Gegenteil. Statt als Held des Protestantismus wahrgenommen zu werden, sahen immer mehr Engländer in der in seinem Namen durchgeführten Politik eine Ursache für die Abkehr Englands vom wahren Glauben: Es stand Jakob und Karl frei, ob sie in ihrer Selbstdarstellung auf die Offenbarung des Johannes oder aber die lex dei im Alten Testament rekurrierten oder nicht. Sobald sie aber eine bestimmte Interpretation dieser Narrative zur Grundlage ihrer Politik erklärten, hatten sie sich der Logik der Mustererzählung zu unterwerfen, oder aber aufgrund der erkennbaren Widersprüche Kritik zu gewärtigen.

Die Reaktionen in England auf die Imagepolitik der beiden Stuartkönige Jakob und Karl vermitteln einen Eindruck von den möglichen politischen Folgen biblizistischer Rhetorik. Damit ist zugleich die Grundsatzfrage berührt, welche Bedeutung dem politischen Biblizismus am Ausbruch des Bürgerkrieges in Schottland und England zuzuschreiben ist. Es versteht sich von selbst, daß hier keine eindeutigen Kausalitäten postuliert werden können. Sicherlich war der Bürger- 
krieg keine notwendige Konsequenz einer biblizistisch vorgetragenen Herrschaftskritik. Wohl aber lassen sich einige Implikationen benennen, die eine biblizistische, an den Normen der lex dei orientierte Weltdeutung mit sich brachte und in bestimmten Situationen eine Entscheidung für eine militärische Auseinandersetzung gegen den König nahelegte. Urheber biblizistischer Sprechakte evozierten mit ihrer am Wort Gottes orientierten Rhetorik, daß sie sich dem Ziel einer Königsherrschaft Gottes auf Erden verbunden fühlten. Über die Frage, wann dieses Ziel auf Erden erreicht sei, gingen die Positionen allerdings diametral auseinander.

In den Augen der Fürsprecher des divine right of kings hatte sich die Königsherrschaft Gottes gleichsam in der Errichtung der Monarchie materialisiert und institutionalisiert. Der König übernahm im Auftrag Gottes die Herrschaftsrolle, die mit oberster Autorität über Staat und Kirche ausgestattet und für die Untertanen unantastbar war. Die Monarchen waren zugleich Werkzeuge Gottes im Heilsplan bis zum Jüngsten Gericht. Mehrere Autoren betonten die besondere Rolle, die den Königen in der Johannesoffenbarung bei der Zerstörung Babylons zugeschrieben wurde. Die Gemeinschaft dieser Könige mit Babylon vor dessen Ende war hierfür kein Gegenargument, sondern nur Teil des Heilsplanes. Götzendienst sei daher kein Grund, die Königsherrschaft in Frage zu stellen. Dies gelte auch für alle anderen Verfehlungen der Könige im Amt. Da die Könige von den Apologeten des divine right of kings als Werkzeuge Gottes verstanden werden, trifft dies in deren Augen auch für deren „Verfehlungen“ zu, die als Gottesstrafe für ein sündhaftes Volk gedeutet werden. Diese politische Theologie monopolisiert den Willen Gottes als Argument in der Hand des Monarchen. Gemäß dem providenziellen Argument kann niemand - außer Gott - den Vorwurf erheben, daß der König gegen die Normen Gottes verstoßen habe.

Eine völlig unterschiedliche Konzeption einer Königsherrschaft Gottes auf Erden vertraten all diejenigen, die den Bund Gottes mit seinem Volk Israel zur Grundlage ihrer politischen Theologie erhoben hatten. Gemäß der Vorstellung eines konditionierten Bundes kann nur diejenige Gemeinschaft Gottes Segen für sich erhoffen, die alle von Gott diktierten Normen minutiös einhielt: Der Verzicht auf jeglichen Götzendienst genoß dabei stets höchste Priorität. Die Heilsgemeinschaft und die politische Gemeinschaft verschmolzen idealiter zu einem einheitlichen Gottesvolk, das Gottes Gesetze achtete und dem daher das Heil gewährt wurde. Dem König kommt in dieser Gemeinschaft die Aufgabe zu, die Einhaltung der göttlichen Normen zu überwachen und sicherzustellen. Sofern er dieser Aufgabe nachkommt, kann er den Gehorsam der Gemeinschaft für sich einfordern.

Diese Vorstellung gehörte für große Teile des schottischen Klerus zum politischen Weltbild und prägte die Wahrnehmung der Königsherrschaft Jakobs VI. und Karls I. Aber auch in England war diese Haltung zur Monarchie sowohl in Teilen des Klerus als auch unter zahlreichen Abgeordneten des Parlaments verbreitet. Für die politische Stabilität der Stuartmonarchie blieb diese politische Theologie solange ohne Folgen, solange die Auffassung vorherrschte, der König 
komme der Aufgabe nach, die Einhaltung der Gesetze Gottes zu überwachen. Sobald sich allerdings bei einflußreichen Kreisen in Kirche und Parlament der Eindruck verfestigen sollte, daß die Übereinstimmung der Monarchie der Stuarts mit der Königsherrschaft Gottes nicht mehr gegeben sei, ja daß die Politik des Königs Verstößen gegen die lex dei Vorschub leiste, war das Potential gegeben, dem König im Namen der Loyalität zu Gott den Gehorsam aufzukündigen.

In den kritischen Sprechakten, die in Schottland als Reaktion auf die Artikel von Perth seit 1618 und in England im Zusammenhang mit der Friedens- und Ausgleichspolitik mit Spanien seit Beginn der 1620er Jahre zu vernehmen waren, diagnostizierten zahlreiche Sprecher ein Auseinanderdriften von göttlichem Normenkatalog einerseits und der Politik des Königs andererseits. Damit läuteten die Kritiker zwar nicht den Bürgerkrieg ein. Sie schufen aber in ihren Mahnreden ein Szenario der Gefährdung Englands durch die Abwendung Gottes von seinem „auserwählten Volk“ und dessen Bestrafung. Zahlreiche Exempla des Alten Testaments lieferten in ihren Predigten und Traktaten Anschauungsmaterial, was Schottland und England zu befürchten hätten, sollte der politische Kurs nicht aufgehalten werden. Die Frage nach den Grenzen der Loyalität gegenüber Jakob VI./I. und Karl I. wurde in diesen Sprechakten in den 1620er Jahren noch ausgespart. Allerdings fanden biblische Gestalten wie Pinhas als Vorbilder Eingang in die Predigten und Traktate, also Personen, die sich durch besonderen Eifer für das Gesetz Gottes auszeichneten und auch vor Mord nicht zurückschreckten, um die göttliche Ordnung wieder herzustellen.

Zwar sollten sich die Wogen zu Beginn der 1630er Jahre sowohl in Schottland als auch in England noch einmal glätten. Die Diskrepanz zwischen der von Karl I. vorangetriebenen Religionspolitik einerseits und dem Ideal einer vollständig reformierten und damit den Gesetzen Gottes entsprechenden Kirche bestimmte aber weiterhin die Wahrnehmung zahlreicher Zeitgenossen. Und im Rahmen dieser Wahrnehmung rückte beinahe zwangsläufig die Frage in den Mittelpunkt, welchen Gesetzen zu gehorchen sei, wenn die königlichen Anweisungen zur lex dei in Widerspruch standen. Die biblizistischen Sprechakte taten dabei das ihre, um diesen Widerspruch stets aufs neue anzuprangern und damit unter den Zeitgenossen präsent zu halten. Manche Autoren wie der schottische Theologe George Gillespie erinnerten die Zeitgenossen ferner daran, daß sie Gott größeren Gehorsam schuldig seien als dem König. Doch war an diesem Punkt eine Grenze erreicht. Gillespie konstatierte wie andere Kritiker der Politik Karls I., daß die Zustände im eigenen Land einem Abfall von Gottes Gesetz gleichkämen und daher eine Umkehr notwendig sei. Sie zogen aber nicht die Konsequenz, ihre Mitstreiter zur offenen Rebellion aufzurufen.

Gleichwohl war der Aufstand der schottischen Covenanters gegen Karl I. letztlich die politische Folge der von der Kanzel und in Traktaten gelieferten $\mathrm{Zu}$ standsbeschreibung des Landes. Er entzündete sich am Versuch der Einführung des englischen Book of Common Prayer, also an einer Maßnahme, die aus Sicht der Presbyterianer die schottische Kirk noch tiefer in den „Götzendienst“ gezwungen und damit vom Zustand der von Gott eingerichteten Kirche entfernt 
hätte. Die Rebellion war der Auftakt zur Umkehr, deren Notwendigkeit zahlreiche Geistliche unter Verweis auf viele Beispiele des Alten Testaments immer wieder bekräftigten. Der Aufstand mündete im National Covenant, einer Bekräftigung des Bundes mit Gott, der den Schotten einen Treueschwur gegenüber Gott und seinen Gesetzen abverlangte, womit sie ihre Loyalität zu Karl I. faktisch aufkündigten. Die lex dei war in diesem Akt der Vergemeinschaftung oberste handlungsleitende Norm. Karl I. sicherten die Unterzeichner nur dann ihre Loyalität $\mathrm{zu}$, sofern er sich seinerseits der lex dei unterwarf, was im Verständnis der Covenanters nicht weniger bedeutete, als vierzig Jahren Religionspolitik der Stuarts in Schottland abzuschwören. Erst nachdem Karl diese Forderungen ablehnte und ein Bürgerkrieg in Schottland unausweichlich war, vergrößerte sich das Arsenal der Argumente, mit denen die Covenanters die Abkehr von ihrem König begründeten: Zusätzlich zur lex dei beriefen sich die Aufständischen nun auch auf das Notwehrrecht sowie das ciceronianische Leitprinzip, daß das Heil des Volkes das oberste Gesetz des Landes sei.

In England fehlte es seit den 1620er Jahren ebenfalls nicht an Sprechakten, in denen Geistliche, Autoren und Abgeordnete des Parlaments eine Diskrepanz zwischen der Einhaltung der Gesetze Gottes und der Friedenspolitik Jakobs I. konstatierten. Nach der Thronbesteigung Karls I. verschob sich der Gegenstand der Kritik von der Außenpolitik des Vorgängers zur Religionspolitik Karls I. Im Zentrum der Kritik standen insbesondere prominente Geistliche wie Richard Montagu oder der spätere Erzbischof von Canterbury, William Laud, die pauschal als Arminians diskreditiert wurden und denen die Kritiker unterstellten, eine heimliche Wiederannäherung der englischen an die römisch-katholische Kirche zu betreiben und damit dem Antichristen in England Tür und Tor zu öffnen. Die stärkere Betonung der ceremonies in der Kirche erschien den Kritikern als Ausweitung des „Götzendienstes“, was der Musterzählung von der Dringlichkeit der Umkehr und der damit verbundenen Einhaltung der Gesetze Gottes neue Nahrung bot. Diese Botschaft dürfte auch im Unterhaus gehört worden sein. Der Abgeordnete John Pym setzte bereits in den Parlamenten der ausgehenden 1620er Jahre alles daran, die Religionspolitik Karls I. zu bekämpfen, indem er versuchte, herausgehobene Protagonisten wie Richard Montagu vom Parlament verurteilen zu lassen. Im Long Parliament von 1640 zählte er dann zu den einflußreichsten Abgeordneten und setzte seine ganze Energie dafür ein, daß das Narrativ der Umkehr und die Notwendigkeit einer Erneuerung des Bundes Englands mit Gott im Parlament Wirkung entfalten konnte. Die zahlreichen Fastenpredigten taten das ihre, die Notwendigkeit zur Reform der Kirche sowie zur Bestrafung all derjenigen, die sich angeblich des „Götzendienstes“ schuldig gemacht hatten, zu unterstreichen.

Auch in den Fastenpredigten findet sich nicht die ausdrückliche Aufforderung zu einem Aufstand gegen den König. Die Prediger in St. Margaret präsentierten den Abgeordneten aber in zahlreichen Predigten biblische Erzählungen und Vorbilder, in denen ausschließlich der bedingungslose Kampf für Gott und gegen die Glaubensfeinde zum Verhaltensideal erhoben wurde. Das Unterhaus sollte sich 
darin beweisen, Eifer für das Gesetz Gottes zu zeigen, deren Widersacher zu vernichten und den Kampf mit der Hure Babylon entschlossen aufzunehmen. Die hierbei häufig zitieren Exempla waren bereits in den Traktaten und Predigten der 1620er Jahren enthalten, in denen manche Autoren zum holy war gegen Spanien und das Papsttum aufriefen. Der Heilige Krieg, von dem die Prediger die Abgeordneten des Long Parliament zu überzeugen suchten, richtete sich dagegen nach innen. Im Fadenkreuz standen die Bischöfe und Repräsentanten der unter Anklage stehenden Kirchenpolitik, ferner die katholische Königin des Landes und ihre Umgebung am Hof, und nur mittelbar auch der König selbst, sofern er sich nicht seinerseits dem Kampf gegen die Glaubensfeinde ohne Rückhalt verschreiben sollte. Rücksicht gegenüber konstitutionellen Schranken war in der Logik dieser Erzählungen bereits eine Abkehr vom Auftrag Gottes, Zögern eine Handlung, die Gottesstrafen heraufbeschwor. Vom schuldigen Gehorsam gegenüber dem König war im Zeitraum von 1640 bis 1642 nur in einer einzigen Fastenpredigt vor den Abgeordneten ausdrücklich die Rede. Bezeichnenderweise weigerte sich das Parlament, diese Predigt zum Druck zu autorisieren.

Die Anwälte des divine right of kings waren auch nach 1640 nicht verstummt. Sie fanden sich aber bis zum Ausbruch des Bürgerkrieges alle im Lager des Königs ein. Mochte die Auffassung von der Unantastbarkeit des Königs als Repräsentant Gottes auf Erden in Stuartengland eine weitgeteilte Auffassung gewesen sein: Aufgrund der Ereignisse in Schottland und England seit dem Jahr 1637 hatte sich dieser Konsens offenkundig verflüchtigt. Die zwei Gruppen, die sich im Sommer 1642 mit ihren Armeen gegenüberstanden, hatten nicht nur divergierende Auffassungen über die Rechte des Königs und die des Parlaments. Sie hatten auch zweierlei Ansichten über die Auslegung von Röm 13, über die Unantastbarkeit des Königs und über die Frage, auf welche Weise die lex dei auszulegen und welche politischen Konsequenzen aus ihr zu ziehen seien. Mochten auch auf beiden Seiten des Schlachtfelds Engländer und Protestanten gegeneinander antreten. Die Politische Theologie der Kontrahenten konnte unterschiedlicher nicht sein. Wie in England eine Königsherrschaft Gottes auf Erden zu erringen sei, darüber entschieden fortan die Waffen. Der politische Biblizismus war das Fundament dafür, daß die Kämpfer beider Seiten darauf vertrauten, Gott an ihrer Seite zu haben. 